Journal Club

Editor's Note: These short, critical reviews of recent papers in the Journal, written exclusively by graduate students or postdoctoral fellows, are intended to summarize the important findings of the paper and provide additional insight and commentary. For more information on the format and purpose of the Journal Club, please see http://www.jneurosci.org/misc/ifa_features.shtml.

\title{
Dorsal Root Ganglion Neurons Carrying a P301S Tau Mutation: A Valid In Vitro Model for Screening Drugs against Tauopathies?
}

\author{
Niels Alberts, ${ }^{\star}$ Karlijn Groen, ${ }^{\star}$ Lisette Klein, ${ }^{\star}$ Marek J. Konieczny, ${ }^{\star}$ and Mandy Koopman ${ }^{\star}$ \\ Department of Molecular Neurobiology, Graduate School of Behavioural and Cognitive Neurosciences, University of Groningen and University Medical \\ Center Groningen, 9747 AG Groningen, The Netherlands \\ Review of Mellone et al.
}

The microtubule-binding protein tau is widely expressed in neurons, where it supports the assembly and stabilization of microtubules (Fong et al., 2013; Spillantini and Goedert, 2013). Under normal physiological conditions, tau lacks secondary structure and is highly soluble. However, posttranslational modifications, including hyperphosphorylation and truncations, are associated with the formation of pathological, insoluble tau-containing aggregates (Zhao et al., 2010). These intracellular aggregates are a major hallmark of various neurodegenerative diseases. These diseases, collectively known as tauopathies, include frontotemporal dementia (FTDP-17), corticobasal degeneration, progressive supranuclear palsy, and Alzheimer's disease (Spillantini and Goedert, 2013). While these diseases differ in their clinical manifestations and the neuronal populations affected, all are characterized by hyperphosphorylated tau, a state that renders the protein unable to stabilize microtubules and support axonal transport (Giacobini and Gold, 2013). Although tau

Received Jan. 12, 2014; revised Feb. 19, 2014; accepted Feb. 21, 2014.

We thank U.L.M. Eisel, J.C.V.M. Copray and S.A. Nelemans (University of Groningen) for their useful advice.

*N.A., K.G., L.K., M.J.K., and M.K. contributed equally to this review.

Correspondence should be addressed to Mandy Koopman, Nijen-

borgh 7, 9747 AG Groningen, The Netherlands. E-mail: m.koopman. 7@student.rug.nl.

DOI:10.1523/JNEUROSCI.0135-14.2014

Copyright $\odot 2014$ the authors $\quad 0270-6474 / 14 / 344757-03 \$ 15.00 / 0$ aggregates are associated with neurotoxicity and are considered to be deleterious, the mechanisms underlying these detrimental effects are still under investigation.

Several models that mimic specific aspects of tauopathy have been developed. Although in vivo transgenic models of tauopathies have provided insight into the pathology, candidate drugs that are useful in these models often fail as potential therapeutics in patients (Fong et al., 2013), making the extent of their clinical value unclear. Several in vitro models in which key hallmarks of tau pathology were reproduced have also been developed (Mellone et al., 2013), making it possible to study specific aspects of tau pathology in an isolated setting. These models may provide useful knowledge about tau pathology while limiting interference of other factors. In one in vitro model, expression of mutated, aggregation-prone $(\Delta \mathrm{K} 280)$ tau repeat domains was induced with doxycyclin in neuroblastoma lines (Khlistunova et al., 2007). In a different model, primary neuronal cell cultures were exposed to the ${ }^{306}$ VQIVYK ${ }^{311}$ hexameric peptide, a fragment of the third repeat domain of tau. This hexameric peptide was made cell-permeable by a special arginine tag and consequently selfassembled into fibrils after cell invasion (Zhao et al., 2010).

These in vitro models allow the study of the formation of tau-containing fibrils and aggregates and may be amenable for high-throughput screening of potential therapeutics (Zhao et al., 2010). Their advantages, however, are accompanied by several disadvantages. Although neuroblastoma lines have the advantage of unlimited proliferation and are therefore suitable for screening large numbers of compounds, they may respond differently to genetic modifications or treatments than normal neurons do. This creates a major hurdle for clinical application (Shastry et al., 2001). The application of the hexameric peptide may provide new insights into tau fibrillization. However, the use of the specific hexameric peptides has limitations in the light of drug screening, because only these added peptides aggregate, not endogenous tau. A cellular model that develops tau pathology without external manipulations would be preferable, because it may better mimic pathological processes found in patients. A disadvantage of all current models is that they show no or only inconsistent levels of spontaneous tau pathology. Therefore, new, high-quality in vitro models that mimic human tau dysfunction are needed to gain insights into the processes underlying these diseases and to develop effective treatments.

Mellone et al. (2013) recently created a model of tauopathy using dorsal root ganglion neurons (DRGs) from mice carrying the human P301S mutation of the tau- 
encoding MAPT gene under a Thy1.2 promoter. In humans, the P301S mutation causes FTDP-17, producing an early onset of symptoms (Allen et al., 2002). Half of the DRGs taken from 3-month-old mice containing human tau had spontaneous tau hyperphosphorylation after 8 weeks in culture. The tau pathology observed in cultured DRGs retained the characteristics and mimicked the symptoms that are found in the brains of both transgenic mice and patients. The pathology was demonstrated using antibodies AT8, AT100, and PHF-1, which are directed against different hyperphosphorylated epitopes of tau, and with the HT7 antibody, which recognizes human tau. DRGs cultured from 1-, 3-, and 5-month-old mice showed increased levels of AT8 and AT100 staining, which further increased without other external manipulations by 8 weeks in culture. In addition, $\mathrm{HT}^{+}$ DRGs had abnormal axons and decreased mitochondrial transport similar to those seen in human tau pathology. AT8 ${ }^{+}$ $\mathrm{HT}^{+}{ }^{+}$DRGs had a shorter survival time than AT8 ${ }^{-} \mathrm{HT}^{+}{ }^{+}$DRGs. Moreover, AT8 labeling appeared to be the earliest sign of tau pathology in this in vitro model, suggesting that AT8 labeling can be used as a fast read-out for tau hyperphosphorylation. Finally, paired helical filaments were detected by MC-1 staining in the DRGmodel (Mellone et al., 2013). Therefore, the DRG model may provide a means by which hyperphosphorylation and fibrillization of tau can be studied to elucidate the pathological process of tau pathology and to screen effects of novel drugs.

Despite the usefulness of the mouse DRG model developed by Mellone et al. (2013), appropriate human-derived cell culture models might resemble human tau pathology more closely. Fong et al. (2013) recently illustrated the applicability of induced pluripotent stem (iPS) cells in disease modeling and drug screening for tauopathies. In this model, iPS cells derived from an individual carrying an A152T mutation of the MAPT gene were used to generate neurons. With specific zinc finger nucleases, Fong et al. (2013) induced site-specific double-stranded DNA breaks, replacing the mutant allele with a wild-type nucleotide sequence. Using this technique, isogenic lines were created that contained one of three different gene doses: zero, one, or two copies of the A152T mutation. The A152A/T cells containing one copy showed increased amounts of axonal degeneration, AT8 ${ }^{+}$ staining, and caspase-3-dependent tau fragmentation. These effects were in- creased even further in the A152T/T cell line, containing two copies of the mutated gene.

Like previously developed cellular models of tauopathies, those developed by Mellone et al. (2013) and Fong et al. (2013) have both advantages and disadvantages. The clear advantage of iPS cells is the possibility of using human cells as a model. The use of iPS cells may be superior to the DRG model because it provides the opportunity to develop patient-specific treatments by taking patients' genetic background into account. However, although the genetics of the iPS-derived neurons may be identical to the cells being modeled, the epigenome of these cells may still differ. Moreover, the model of Fong et al. (2013) requires zinc finger nucleases to ensure genetic controllability and to generate control groups. The DRG-model of Mellone et al. (2013) does not require additional techniques to create control groups. Therefore, this model might be more useful for large scale, high-throughput screening in early stages of drug development. After all, identifying novel, effective drugs for tauopathies is of major importance given that no effective treatments are yet available (Giacobini and Gold, 2013).

A drawback of the DRG model is that these sensory neurons are not usually implicated in tauopathies. Moreover, only $50 \%$ of the cultured $\mathrm{HT}^{+}{ }^{+}$DRGs had hyperphosphorylation, as shown by the AT8 staining (Mellone et al., 2013). However, the increasing number of $\mathrm{HT}^{+}{ }^{+} \mathrm{AT} 8{ }^{+}$ DRGs implies that the pathology progresses over time and ultimately more of the DRGs may be affected. Hence, the progression of tau hyperphosporylation in DRGs may resemble human pathology in this aspect. Moreover, DRGs are well suited for studying axonal outgrowth, guidance, and stability. Therefore, they might be helpful in studying cytoskeletal integrity, which is highly associated with tau-dysfunction (Stoppelkamp et al., 2011). In addition, the ability to culture the DRGs for a relatively long period compared with, for example, cortical neurons, enables assessment of the effects of pharmaceutical compounds on the formation of paired helical filaments and aggregates over a timespan of several weeks. Targeted disruption of specific steps in the formation of aggregates and filaments in such longitudinal studies may provide insight in the question of which stage of the aggregation formation of tau fibrils is most neurotoxic.

As noted above, the DRG model will likely prove useful. Nonetheless, altera- tions based on previous research might further improve its value in drug screening. There are indications that endogenous murine tau interferes with transgenic, human tau during the development of tau pathology. Studies report either accelerated human tau aggregation when murine tau is absent (Ando et al., 2011) or an increase of human hyperphosphorylated tau and neurofibrillary tangle formation in the presence of murine tau (BagliettoVargas et al., 2014). Therefore, knocking out murine tau in the P301S-background might further improve the representative value of the DRG model. Moreover, as illustrated by Fong et al. (2013) regulation of tau assembly and hyperphosphorylation are gene-dose-dependent. Extending the transgenic lines with a variety of P301S-htau insertions may therefore provide a way of studying this concentration effect at the level of DRGs. Furthermore, since tau truncations are observed in tauopathies (Zhao et al., 2010), it would be interesting to test whether tau is also fragmented in the DRG model.

In conclusion, Mellone et al. (2013) have created a novel cell model for tau pathology in which tau hyperphosphorylation and aggregation develop without external manipulation. DRG neurons may not be as long-lived as neuroblastoma cells, but they appear to be more similar to the neurons that should eventually be targeted in the clinic. The use of patient-derived iPS cells provides an unprecedented tool for studying human tau pathology, but it certainly does not exclude the use of the DRG model. Although DRGs are not usually affected in tauopathies, Mellone et al. (2013) clearly demonstrate that the model resembles the development of pathology found in human tauopathies. The ability to culture the DRGs for 8 weeks makes this model a useful tool in the testing and development of drugs against tauopathies and to study the underlying mechanisms of tau pathology.

\section{References}

Allen B, Ingram E, Takao M, Smith MJ, Jakes R, Virdee K, Yoshida H, Holzer M, Craxton M, Emson PC, Atzori C, Migheli A, Crowther RA, Ghetti B, Spillantini MG, Goedert M (2002) Abundant tau filaments and nonapoptotic neurodegeneration in transgenic mice expressing human P301S tau protein. J Neurosci 22:9340-9351. Medline

Ando K, Leroy K, Héraud C, Yilmaz Z, Authelet M, Suain V, De Decker R, Brion JP (2011) Accelerated human mutant tau aggregation by knocking out murine tau in a transgenic mouse model. Am J Pathol 178:803-816. CrossRef Medline

Baglietto-Vargas D, Rodriguez-Ortiz CJ, Ki- 
tazawa M, Medeiros R, Le EJ, Green KN, Estrada-Hernandez T, LaFerla FM (2014) Endogenous murine tau promotes neurofibrillary tangles in $3 \times \mathrm{Tg}$-AD mice without affecting cognition. Neurobiol Dis 62:407-415. CrossRef Medline

Fong H, Wang C, Knoferle J, Walker D, Balestra ME, Tong LM, Leung L, Ring KL, Seeley WW, Karydas A, Kshirsagar MA, Boxer AL, Kosik KS, Miller BL, Huang Y (2013) Genetic correction of tauopathy phenotypes in neurons derived from human induced pluripotent stem cells. Stem Cell Reports 1:226-234. CrossRef Medline

Giacobini E, Gold G (2013) Alzheimer disease therapy-moving from amyloid- $\beta$ to tau. Nat Rev Neurol 9:677-686. CrossRef Medline
Khlistunova I, Pickhardt M, Biernat J, Wang Y, Mandelkow EM, Mandelkow E (2007) Inhibition of tau aggregation in cell models of tauopathy. Curr Alzheimer Res 4:544-546. CrossRef Medline

Mellone M, Kestoras D, Andrews MR, Dassie E, Crowther RA, Stokin GB, Tinsley J, Horne G, Goedert M, Tolkovsky AM, Spillantini MG (2013) Tau pathology is present in vivo and develops in vitro in sensory neurons from human P301S tau transgenic mice: a system for screening drugs against tauopathies. J Neurosci 33:18175-18189. CrossRef Medline

Shastry P, Basu A, Rajadhyaksha MS (2001) Neuroblastoma cell lines - a versatile in vitro model in neurobiology. Int J Neurosci 108: 109-126. CrossRef Medline
Spillantini MG, Goedert M (2013) Tau pathology and neurodegeneration. Lancet Neurol 12:609-622. CrossRef Medline

Stoppelkamp S, Bell HS, Palacios-Filardo J, Shewan DA, Riedel G, Platt B (2011) In vitro modelling of Alzheimer's disease: degeneration and cell death induced by viral delivery of amyloid and tau. Exp Neurol 229:226-237. CrossRef Medline

Zhao K, Ippolito G, Wang L, Price V, Kim MH, Cornwell G, Fulenchek S, Breen GA, Goux WJ, D'Mello SR (2010) Neuron-selective toxicity of tau peptide in a cell culture model of neurodegenerative tauopathy: essential role for aggregation in neurotoxicity. J Neurosci Res 88:3399-3413. CrossRef Medline 